\title{
Efecto de la anemia ferropriva en el lactante sobre el desarrollo psicológico del escolar
}

\author{
Francișca Rivera A. ${ }^{\text {; }}$ Tomás Walter $\mathrm{K} .^{2}$ \\ Effects on school performance at age ten years of \\ former iron deficiency anemia in infancy
}

\begin{abstract}
Iron deficiency aremio is the most prevolent nutriticnal disorder in the world. Its effects are associoled with ceaved psychomotor development and behavior changes on whose 'ong ierm persislence there stl remains uncertcinty. Two sludies in children aged 5 years hove s'own continuing lower performances in those with "crmer anemia. To evaluate ihe long term. effecls at age 10 veais in the same cohort previously exarrined al age 5 years, we evaluated 64 children. 34 with former anemia and 30 without it. A complete psychological assessmert didn'l show the differerces observed al 5 years in intelleclual $\|$ Q of those without ontecedenil of aremio in ieaction ime, scholastic achievement abilities iwritten tanguage and specific skills\}, scholar achievement iphysicol exucation onc mean gerieral schocl grodes), and ir the scholar behavior. These results suggest thal the association belween anem a in infancy and negaive effects on development continues a 10 years of age, shown mainly at specific behaviours required in the school context.
\end{abstract}

(Koy words: anemia, iron deficiency, schoo' performance.)

La anemia por deficiencia de hierro constituye el déficit nutricional de mayor prevalencia en la población mundial, situación más acentuada en países de menor desarrollo por la dieta pobre en hierro biodisponible. Por sus altos requerimientos y una dieta relativamente pobre en hierro, la lactancia (6 a 24 meses de edad) es el período más susceptible al déficit de hierro. En Chile, en poblaciones de niveles socioeconómicos bajos, la anemia por deficiencia de hierro se detecta en $25 \%$ a $45 \%$ de los lactantes de esa edad, etapa que corresponde a la de mayor vulnerabilidad del sistema nervioso central ${ }^{1-3}$.

La anemia ferropriva en el periodo de la lactancia se asocia con desarrollo psicomotor inferior, diferencia que -aunque leve- es consistente ${ }^{4-8}$. Los estudios sobre la persistencia de

1. Psicóloga Unidad de Neurofisiología y Psicología del Desarrollo. Instituto de Nutrición y Tecnología de los Alimentos (INTA). Universidad de Chile.

2. Médico. Unidad de Hematologia, Instituto de Nutrición y Tecnología de los Alimentos (INTA), Universidad de Chile.

Trabajo finacciado por Nestle Foundation, Lausana, Suiza, y Fondecyt, Chile (proyecto N 1930810). los efectos de la anemia, una vez tratada, sobre el desartollo psicológico en el corto y largo plazo, muestran que persisten las diferencias en desmedro de los niños con el antecedente ${ }^{4-10}$. Sin embargo, estos resultados son objeto de controversias teóricas y metodológicas. En Chile y Costa Rica se ha demostrado que, cuando la anemia ocurre durante los primeros dos años de vida, sus efectos comprometen el desarrollo psicomotor y conductual del lactante, aun después de tratamiento con hierro oral de tres meses de duracion, suficiente para corregir la anemia y la mayoría de las mediciones hematológicas ${ }^{4,5,8}$. La asociación entre anemia ferropriva a la edad de un año y desarrollo, persiste a los 5 años de edad en lo referente a habilidades cognitivas, motoras y conductuales ${ }^{9-11}$. Por otra parte, en lactantes de Indonesia se mostró que, si bien la anemia por deficiencia de hierro afectaba al desarrollo psicomotor, el efecto revertía con una terapia de cuatro meses ${ }^{12}$. Aun cuando los estudios de seguimiento revelan la persistencia de los efectos de la anemia por deficiencia de hiero a la edad de un año, se cuestiona que en la etapa preescolar la relación causa a efecto tenga una relación lineal, proponiéndose considerar a 
la afección como un factor de riesgo para el desarrollo psicológico, cuyo mecanismo de acción aún se desconoce.

E1 análisis que se ofrece a continuación forma parte de un estudio de seguimiento de una cohorte de niños con y sin antecedentes de anemia por déficit de hierto en el primer año de vida, que fueron caracterizados entonces por variables dietarias, antropométricas, de morbilidad y demograficas. Representa un corte transversal a los diez años de edad. Se trata de estudio exploratorio, asociativo, cuyo objetivo es evaluar a largo plazo el efecto de la anemia temprana sobre el desartollo psicológico de niños en edad escolar.

\section{Material y Método}

En los años comprendidas entre 1982 y 1986 las unidades de hematología, neurofisiología y psicologia del desarrollo del Instituto de Nutrición y Tecnologia de los Alimentos de la Unjyersidad de Chile (INTA) realizaron un estudio en terreno de fortificación de los nucrientes con hierro, que incluyó 500 lactantes del área Sur-Oriente de la ciudad de Santiago. La muestra se obtuvo de una población urbana de condiciones socioeconómicas bajas, homogenea en sus caracteristicas socioculturales y etnicas. Los criterios de selección fueron: peso de nacimiento $>2500 \mathrm{~g}$ y ausencia de patologías genéticas, neutológicas o metabólicas. Los niños fueron controlados periódicamente en el consultorio de salud de la comunidad. A los 12 meses de edad se realizaron evaluaciones hematológica y psicológica a 240 lactantes de la muestra. que se repitieron luego de tres meses de tratamiento con hierro oral. En este subgrupo se identificaron: 58 niños normales $(\mathrm{Hgb}>$ $11,0 \mathrm{~g} / \mathrm{dl}$, volumen corpuscular medio < $70 \mathrm{hl}$, saturación de la transfertina $10 \%$, protoporfirina libre eritrocitaria < $100 \mu \mathrm{g} \mathrm{ZPP/dl} \mathrm{GR,} \mathrm{ferritina} \mathrm{sérica} \mathrm{<} 1(\mathrm{lng} / \mathrm{m})$ ): 127 tirnos sideropénicos $(\mathrm{Hg} b>11 \mathrm{~g} / \mathrm{dl}$, y con alteración de al menos un parámetro hematológico) y 55 nî̃os anémicos $(\mathrm{Hgb}<$ $11.0 \mathrm{~g} / \mathrm{dl}$ y alteración de 2 a más parámetros bioquí$\operatorname{micos})^{4.8}$.

Posteriormente, a la edad de 5 aflos, se realizo un seguimiento de los nijos con y sin antecedentes de anemia (4) y 29 respectivamente), que contemplo evaluaciones médica, psicológica y social completas, excluyéndose los que solo tenían sideropenia por no mosirar éstos diferencias en el rendimiento psicomotor con los sin antecedentes de anemia, y como un modo de enfocar la atención en los casos extremos con respecto a la nutrición de hierro". De los 70 niños evaluados a los 5 años de edad se recuperaron 64 para el presente estudio $(95,7 \%), 34$ con antecedente de anemia ferropénica (A) y 30 con un estado nutricional de hierro nomal (NA) al año de edad. Cuatro casos se perdieron por cambio de domicilio y dos por rechazo del protocolo (inasistencia a tas citaciones). De los 64 niños incorporados, no todos completaron el total de las evaluaciones. debido fundamentalmente a asistencia irre- gulat a las cilaciones. cuyos motivos no fue posible investigar. La edad promedio de estos ninos fue de I0 antos I mes y la escolaridad promedio de 4 anos, no existiendo diferencias significativas entre casos y cuntroles respecto a ellas.

Los sujetos que confornan la muestra fucron invitados a participar en este estudio cuando cumplieron la edad de 10 años por intermedio de la misma enfermera de terteno que los había citado a los 5 años. Una ver. que los padres firmaron un consentimiento infonnado. los niños asistieron a tres sesiones de evaluación, de aproximadamente 1,5 horas cada una, con un intermedio de descanso. Las evaluaciones realizadas fueron: 1) capacidad intelectual, mediante la escala de L. Terman y M. Merrill, forma L-M, utilizíndose el análisis de seis factores ptopuestos por $\mathrm{R}$. Valett (comprensión general, habilidad visomotora, razonamiento aritmético, memoria y concentración, vocabulario $y$ fluidez verbal $y$ juicio y razonemiento ${ }^{13}$ : 2) motricidad, aplicando la prueba de destrezas motoras de $R$. Bruitinks y Oseretsky, para motricidad gruesa y fina ${ }^{14}$, 3) integración visomotora, mediante la prueba de K. Beery is. 4) habilidades psicoeducacionales, empleando la batería de R. W. Woodcock, que ofrece un indicador global respecto al rendimienta en tareas que miden una amplia variedad de habilidades cognitivas y lambién cualmo indicadores de comportamiento en habilidades de aprovechamiento escolar (lectura, matemáticas, lenguaje escrito y destrezas específiças requeridas para la lectura, ortografía y resolución de problemas aritméricos aplicados $\left.)^{(t ;} ; 5\right)$ autoestima. evaJuada con la escala de Piers-Harris que lo hace a partir de seis factores (conducta, estado intelectual y académico, apariencia y atributos físicos, ansiedad, popularidad y sitisfaccion) ${ }^{17}$; 6) presencia de problemas conductuales, aplicando el inventario de destrezas sociales y problemas conductuales de T. Achenbach ${ }^{18} ;$ 7) rendinjento y comportamienı cscolar, mediante una guía diseñada específicamenle para registrar, en basc al informe del profesor jefe. el rendimiento promedio del niño respecto a su curso en las asignaturas de castellano, aritmélica y educación física, el promedio general de notas del último año cursado con respecto a sus pares, y sus característicos en relación a sus compañeros, al trabajo y a la educadora: 8 ) calidad de la estimulación del hogar, evaluada con el inventario HOME de B. Caldwell y R. Bradley para familias de adolescentes tempranos y que explora seis áreas (ambiente físico, material de aprendizaje, modelos, actividades for. mativas, variedad de experiencias y aceptación y respeto por el escolar): 9) Desarrollo neurológico, evaluado con uл examen clinico por un especialista en base a una paula de rastreo elaborada y utilizada por los neurologos de la unidad de genética y enfermedades metabólicas del INT $A ; \mid 0$ ) salud física, antropometría y desarrollo puberal, mediante examen clínico y de acuerdo con los criterios del Centro Estadounidense de Estadisticas de Salud (NCHS) y los de Tanner;11) estado nutricional de hierro. utilizando la concentración de hemoglobina, el volumen corpuscular medio y la saruración de la transferina (se consideró anemia la concentración de hemoglobina menor a II gfdl, o la alteración de al menos dos de los eriterios antes mencionados. medidos en sangre obtenida pcr punción venosa): (2) ca. pacidad intelectual de la madre, evaluada con la escala para medición de lá inteligencia en adulıos de Weschler (abreviada)19; 13, síntomas depresivos de la madre, eva- 
luada con la Escala CES-D que indaga la frecuencia y duración de síntomas cognitivos, afectivos, conductuales y somáticos asociados a depresión ${ }^{20}$. Todas las evaluaciones fueron realizadas con los mismos instrumertos aplicados a los 5 años de edad.

Las mediciones psicológicas del niño como de la madre fuecon efectuadas por una psicóloga previamente familiarizada en el uso de los instrumentos. Las evaluaciones es. colares fueron realizadas por una psicopedagoga: las médicas, por especialistas en pediatrfa o neurologia; la an Iropométrica y la obtención de muestra de sangre, por una enfermera. En todos los casos se aplico el método de doble ciego a la condición de anemia del niño al año de edad.

Para analizar estadísticamente los datos se utilizó la prueba $F$ para comparar diferencias de promedio entre grupos independientes para distribuciones normales de variables continuas (capacidad intelectual. motricidad, integración visomotora, depresión materna). En caso de obrenet una distribución que no fuera normal, se aplicó la prueba de Kruskal-Wallis. En las mediciones no expresadas en una medida estándar, como la edad equivalente en las habilidades psicoeducativas y el rendimiento en notas de niños que asisten a establecimientos educacionales distintos, se calculó, en el primer caso, la diferencia entre las edades cronologica y equivalente para cada nifio, de manera de controlar la diferencia de edad entre los niños; y en el segundo, la diferencia entre el rendimiento promedio del curso y el promedio del niño en cada caso para controlar la variedad de establecimiento educacional y saber con mejor aproximación la posición del rendimiento del niño con respecto al de su curso. De este modo, un valor numérico menor corresponde a un mejor rendimiento (Ejemplo: pronxdio general de notas del curso $=5.6$ : promedio general de notas del niño $=5,8$ : diferencia $5,6-5,8=-0,2$ ). Para comparar distribuciones de frecuencias en variables nominales se utilizo la prueba de chi cuadrado. Para estimar la fuerza de asociación entre un conjunto de variables y cada variable psicológica dependiente se aplicó un cólculo de regresión múltiple. Para todas las pruebas estadísticas se estableció el nivel de significación $\mathrm{p}$ en 0.05 , y el de error $B$ en 0.2 .

\section{Resultados}

La comparación de la capacidad intelectual (Cl), la motricidad gruesa (CMG), la motricidad fina (CMF), la motricidad global (CMT) e integración visomotora (VMI) no mostró diferencias significativas entre los niños con y sin antecedentes de anemia. Tampoco en los factores evaluados por la escala de inteligencia. En las destrezas motoras no se observó diferencias en las medidas en CMG, CMF y VMI, pero el tiempo de reacción fue más lento en los niños con antecedente de anemia que en los controles $(13,47 \pm 6,84$ ante $11,13 \pm 3,99$, respectivamente, $\mathrm{p}<0,05$ ) (tabla 1). En las habilidades psicoeducativas sóla se observó diferencia significativa en desmedro de los niños con antecedente

\section{Tabla 1}

Rendimiento intelectual y motor en escolares con antecedentes de anemia ferropriva en la lactancia

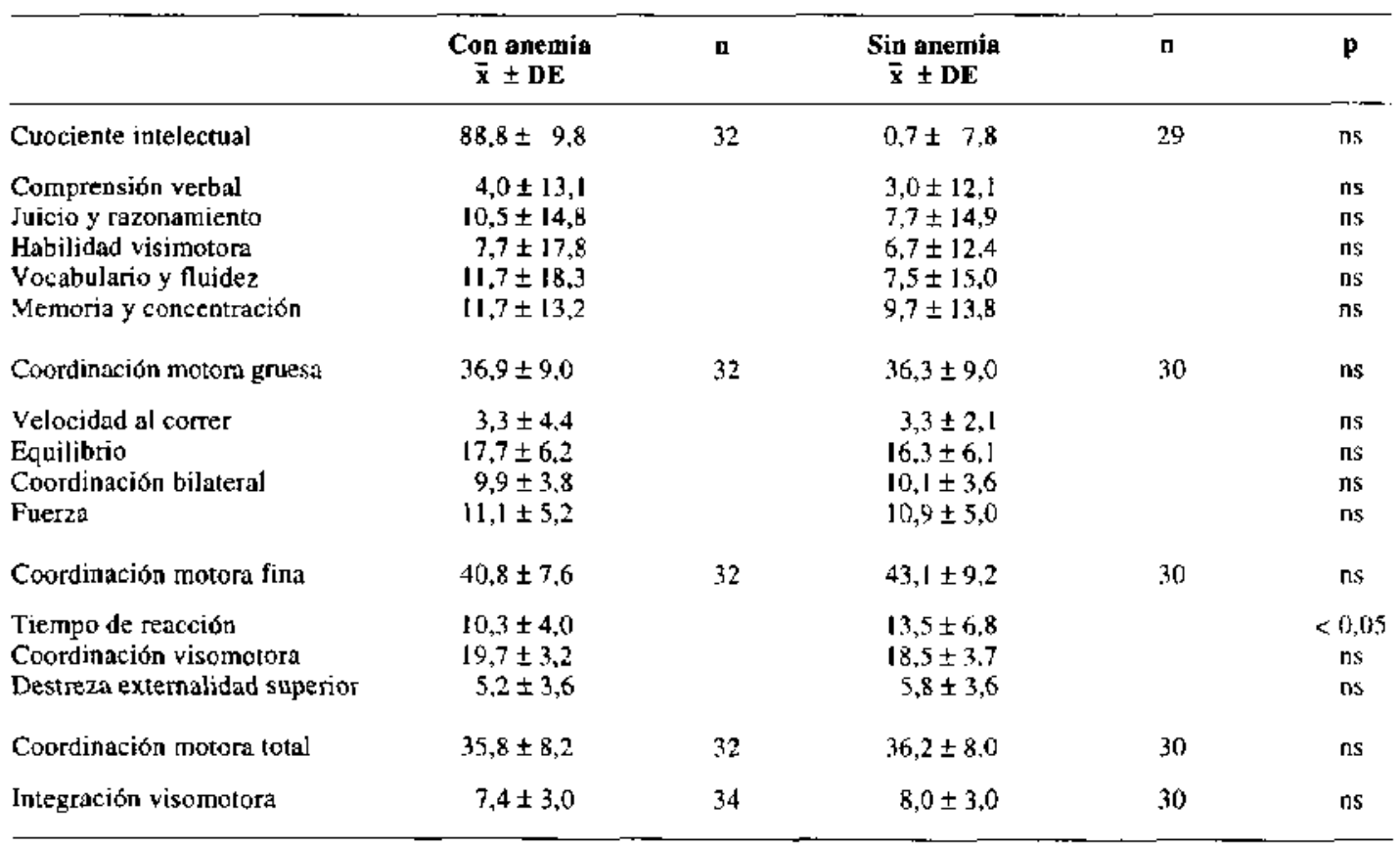


de anemia en las habilidades de aprovechamiento escolar, relacionadas con el lenguaje escrito $(13,58 \pm 19,08$ ante $2,47 \pm 16,01, \mathrm{p}<0,05) ; y$ con destrezas específicas requeridas para la lectura, dictado y resolucion de problemas aritméticos aplicados $(4,03 \pm 23,5$ ante $5,37 \pm 17,65$, $p<0,05$ ). En el rendimiento escolar se registró una diferencia en favor del grupo control en el promedio general de notas $(0,29 \pm 0,61$ y $-0,03$ $\pm 0,49$, p 0,05 ), y en la asignatura de educación física $(0,22 \pm 0,59$ ante $-0,1 \pm 0,44, p<0,05)$ (tabla 2). Las características conductuales y socioemocionales de ambos grupos no registraron diferencias en la autoestima, las destrezas sociales o la presencia de problemas conductuales, pero los niños con antecedentes de anemia solicitaban con mayor frecuencia ayuda en sus trabajos escolares $(\mathrm{p}<0,05)$ y eran más dependientes de la aprobación y apoyo del profesor $(p<0,01)$.

Salud. No se encontraron diferencias en e] desarrollo antropométrico (peso/edad, talla/edad y peso/talla) y el estado nutricional de hierro actual. El desarrollo puberal (Tanner) no excedió la etapa 1, confirmando que todos los niños eran prepúberes, y tampoco se observaron diferencias en el desarrollo neurológico.

Las madres de los niños de ambos grupos no diferían respecto a la presencia de síntomas de depresión y en capacidad intelectual, pero en cambio la calidad de la estimulación brindada en el hogar (HOME) era menor en el grupo con antecedente de anemia $(50,4 \pm 9$ ante $57,7 \pm 7,1$, $\mathrm{p}<0,005$ ) (tabla 3). Las diferencias se centraron en la calidad del ambiente físico, los materiales de aprendizaje, la variedad de experiencias y la aceptación y respeto de los padres hacia el niño.

La fuerza de la asociación específica entre la anemia, la calidad de la estimulación recibida y los rendimientos observados a través del método paso a paso en una regresión múltiple no mostraron asociaciones significativas entre la anemia y la calidad de la estimulación (HOME) con el promedio general de notas, pero sí entre la anemia con el rendimiento en educación física $(p<0,05)$, el lenguaje escrito $(p<0,02)$ y las destrezas en aprovechamiento escolar $(\mathrm{p}<$ $0,05)$; no así entre éstas últimas y el HOME. Sólo se observa una relación significativa entre HOME y tiempo de respuesta $(\mathrm{p}<0,04)$, anulándose la relación con la anemia (tabla 4).

\section{Comentario}

Estos resultados permiten señalar la persistencia de las diferencias en variables psicológicas a los 10 años de edad, entre los niños con y

Tabla 2

Rendimiento en habilidades psicoeducacionales y asignaturas de escolares con antecedentes de anemia ferropriva en la lactancia

\begin{tabular}{|c|c|c|c|c|c|}
\hline & $\begin{array}{c}\text { Con anemia } \\
\overline{\mathbf{x}} \pm \mathbf{D E}\end{array}$ & $\mathbf{n}$ & $\begin{array}{c}\text { Sin anemia } \\
\overline{\boldsymbol{x}} \pm \mathbf{D E}\end{array}$ & $\mathbf{n}$ & $\mathbf{p}$ \\
\hline Habilidades cognilivas & & 33 & & 30 & \\
\hline $\begin{array}{l}\text { Lenguaje oral } \\
\text { Razonamiento } \\
\text { Rapidee percept.-visual }\end{array}$ & $\begin{array}{r}-5,1 \pm 24.4 \\
0.6 \pm 28.1 \\
30,6 \pm 1.3 .4\end{array}$ & & $\begin{array}{l}-7,4 \pm 14,5 \\
-2,0 \pm 24,4 \\
28,5 \pm 16.2\end{array}$ & & $\begin{array}{l}\mathrm{ns} \\
\mathrm{ns} \\
\mathrm{ns}\end{array}$ \\
\hline Aprovechamiento escolar & & 3.3 & & 30 & \\
\hline $\begin{array}{l}\text { Leciura } \\
\text { Matemálicas } \\
\text { Lenguaje escrito } \\
\text { Destrezas }\end{array}$ & $\begin{array}{r}-37.9 \pm 55,7 \\
14,6 \pm J 3.4 \\
13,6 \pm 19.1 \\
4.0 \pm 23.5\end{array}$ & & $\begin{array}{r}-41,8 \pm 45,4 \\
11,0 \pm 10,2 \\
2,5 \pm 16,0 \\
-5,4 \pm 17.7\end{array}$ & & $\begin{aligned} & \text { ns } \\
& n s \\
< & 0.05 \\
< & 0.05\end{aligned}$ \\
\hline Asignaturas & & 26 & & 29 & \\
\hline $\begin{array}{l}\text { Castellano } \\
\text { Matemúticas } \\
\text { Educación fisica } \\
\text { Promedio general }\end{array}$ & $\begin{array}{l}0,1 \pm 0.6 \\
0,3 \pm 1.0 \\
0,2 \pm 0.6 \\
0,3 \pm 0.6\end{array}$ & & $\begin{array}{l}-0,0 \pm 0,8 \\
-0,1 \pm 0,8 \\
-0,1 \pm 0,4 \\
-0,03 \pm 0,5\end{array}$ & & $\begin{aligned} & n s \\
& n s \\
< & 0,05 \\
< & 0,04\end{aligned}$ \\
\hline
\end{tabular}


Tabla 3

Características de las madres y de la calidad de estimulación en escolares con antecedentes de anemia ferropénica durante la lactancia

\begin{tabular}{|c|c|c|c|c|c|}
\hline & $\begin{array}{c}\text { Con anemia } \\
\overline{\bar{x}} \pm \mathrm{DE}\end{array}$ & $\mathbf{n}$ & $\begin{array}{c}\text { Sin anemia } \\
\overline{\mathbf{x}} \pm \mathbf{D E}\end{array}$ & $\mathbf{n}$ & $\mathbf{p}$ \\
\hline Cuociente intelectual & $83,1 \pm 12,9$ & 25 & $81,2 \pm 8,2$ & 23 & ns \\
\hline Estímulos en el hogar & $50,4 \pm 9,0$ & 24 & $57,7 \pm 7,1$ & 23 & $<0,005$ \\
\hline Anbiente físico & $56,7 \pm 1,8$ & & $6,7 \pm 1,0$ & & $<0,03$ \\
\hline Material de apoyo & $4,6 \pm 2,1$ & & $6,8 \pm 1,8$ & & $<0,0009$ \\
\hline Modelos & $9,2 \pm 2,1$ & & $9,7 \pm 1,8$ & & ns \\
\hline Actividad formativa & $16,1 \pm 3.2$ & & $17,7 \pm 3,3$ & & ns \\
\hline Variedad de exposicion & $6,2 \pm 1,5$ & & $7,2 \pm 1,1$ & & $<0,02$ \\
\hline Aceptación y respeto & $8,6 \pm 1,5$ & & $9,6 \pm 1,4$ & & $<0,02$ \\
\hline
\end{tabular}

\section{Tabla 4}

Relación entre anemia, estimulación en el hogar (HOME) y rendimiento en escolares con antecedentes de anemia ferropénica durante la lactancia

\begin{tabular}{lccccc}
\hline & Anemia (t) & P & HOME (T) & P \\
\hline Educación física & $-2,06$ & $<0,05$ & $-0,62$ & $\mathrm{~ns}$ \\
Lenguaje escrito & $-2,43$ & $<0,02$ & $-1,03$ & $\mathrm{~ns}$ \\
Destrezas & $-2,06$ & $<0,05$ & $-0,59$ & $\mathrm{~ns}$ \\
Tiempo de respuesta & 1,12 & $\mathrm{~ns}$ & 2,20 & $<0,05$ \\
\hline
\end{tabular}

sin antecedentes de anemia ferropriva. Si bien no se observaron las diferencias detectadas a los 5 años en habilidades globales ${ }^{4}$, éstas se observaron en habilidades o funciones específicas y el rendimiento escolar, todas en desmedro del grupo con antecedente de anemia.

Las diferencias registradas en el largo plazo sobre ciertas habilidades de aprovechamiento escolar y el rendimiento escolar concuerdan con las de los únicos estudios de características similares en que se también el rendimiento escolar de los niños con antecedentes de anemia por déficit de hierro en la infancia era significativamente menor ${ }^{21}$, así como también en niños mayores y adolescentes ferroprivos ${ }^{22-24}$. En escolares tailandeses se había observado que los que tenfan anemia ferropriva mostraban un rendimiento inferior en tareas escolares relacionadas con lenguaje, mientras las diferencias observadas en las pruebas de matemáticas no alcanzaban el nivel de significación elegido ( $\mathrm{p}=$ $0,08)^{25}$. Estos antecedentes permiten postular que el efecto de la anemia por escasez de hierro (ocurra èsta en etapas tempranas o más tardías del desarrollo) podría asociarse en la etapa escolar con alteraciones de funciones más específicas. En ese sentido, se ha señalado que la deficiencia de hierro tendría efectos adversos, más que en una habilidad mental global, sobre procesos cognitivos determinados ${ }^{26}$. El menor rendimiento escolar de los niños con antecedentes de anemia en educación física podría ser, de alguna manera, inconsistente con la ausencia de diferencia observada en la evaluación de la motricidad gruesa. Sin embargo, es posible que la evaluación de dicha asignatura, por provenir de mediciones repetidas realizadas, supuestamente, por una misma persona durante al menos un año, comprometa otro tipo de variables no consignadas. Aun así. parece ser un área más vulnerable en los niños con antecedentes de la deficiencia nutricional. Esto sería consistente con estudios realizados en ratas anémicas ferroprivas (pero transfundidas hasta conseguir una concentración normal de hemoglobina), que demuestran disminución de la fuerza muscular, reduccion de los movimientos corporales y menoscabo de la energía física ${ }^{27}$. También en modelos animales se ha señalado que las áreas del sistema nervioso relacionadas con el control de la actividad motora son ricas en hierro, de modo que cuando ocurre una anemia en períodos de gran crecimiento y desarrollo cerebral, la restitución del hierro cerebral sería muy lenta, persistiendo el déficit en la edad adulta ${ }^{28}$. Pero pudiese ser que no sólo la anemia ferropriva explicase las diferencias observadas en la acti- 
vidad física, ya que el modelo que ofrecen los padres en actividad física se relaciona con la que muestran sus hijos. El fomento de la actividad física y la predisposición genética a mayor nivel de ésta también podrían participar en la relación que se comenta ${ }^{29}$.

Las diferencias en la conducta como escolares de los niños con antecedentes de anemia por escasez de hierro a esta edad, como solicitar ayuda en su trabajo con mayor frecuencia y una actitud más dependiente del educador, no concuerdan con lo registrado sobre dependencia a la de 5 años ${ }^{11}$. Entonces, se observó que los niños con el antecedente eran más temerosos e inhibidos, en coincidencia con lo que se había visto en ellos durante la anemia ${ }^{4}$. Por otra parte se ha mostrado que lactantes con anemia ferropriva mantienen relaciones de mayor proximidad con su madre y promueven con más frecuencia el contacto corporal con ella ${ }^{30}$. Si bien estos hallazgos no son comparables, por haberse usado diferentes instrumentos para medir el comportamiento, es posible que las conductas a los 10 años de edad tengan algún antecedente en etapas previas del desarrollo. Así, los lactantes más temerosos, con mayor necesidad de cercanía de la madre, podrían desarrollar patrones de relación con mayor dependencia a figuras de autoridad.

En lo que respecta a la influencia de posibles variables intervinientes, potencialmente de confusión, sobre el efecto de la anemia por deficiencia de hierro sobre el rendimiento, no se encontraron las diferencias observadas a los cinco años en las características depresivas de las madres de los niños con el antecedente, pero la calidad de la estimulación recibida por éstos era menor, tal como había ocurrido también a la edad de cinco años ${ }^{9}$. Otros han observado una asociación similar entre HOME y lactantes anémicos, la que no persistía en el seguimiento a los 5 años, ${ }^{5,10}$. Al analizar las contribuciones telativas del HOME y de la anemia en los rendimientos observados, se encontró una asociación significativa entre el HOME y la velocidad del tiempo de respuesta, que hacía desaparecer la relación de éste con la anemia. En cambio persistió una asociación significativa entre antecedente de anemia por déficit de hierro y el rendimiento en educación física, en lenguaje escrito y en destrezas de aprovechamiento escolar, que no se observ6 con el HOME.
El diseño de este estudio, el tamaño de la muestra y la pérdida muestral con respecto a los 12 meses y los 5 años, las mediciones realizadas, los instrumentos utilizados en los distintos momentos de evaluación y la falta de normas estándar para nuestra población, restringen la posibilidad de precisar el papel de la anemia ferropriva en el desarrollo psicológico a largo plazo de niños provenientes de un nivel socioecon6mico bajo. Sin embargo sus resultados sugieren la necesidad de estar alertas a los riesgos que la deficiencia temprana pudiese acarrear al desarrollo integral de las personas.

\section{Resumen}

La anemia por deficiencia de hierro es el trastorno nutricional de mayor prevalencia en el mundo y puede afectar el desarrollo psicomotor y la conducta del niño. No hay certeza de la reversibilidad de sus efectos en el largo plazo. Se realizá un estudio transversal a la edad de 10 años de edad, en una cohorte de 64 escolares de condición sociosconómica baja, 34 de los cuales habian sufrido anemia por deficiencia de hierro alrededor de la edad de un año y $30 \mathrm{sin}$ dicho antecedente. La evaluación psicologica no mostró las diferencias detectadas previamente. a los 5 años de edad, en habilidades cognitivas y motoras globales. Sin embargo, se encontraron diferencias significativas a favor de los niños libres de anemia al año de edad en aprovechamiento escolar (lenguaje escrito y destrezas específicas: $p<0,05$ ): rendimiento escolar (promedio general de notas y educación física: $p<0,05$ ); y en el comportamiento en el ámbito escolar (menor dependencia del profesor: $\mathrm{p}<0,01$; $y$ menor frecuencia de solicitud de ayuda en trabajos escolares: $p<0,05$ ). Los efectos negativos sobre el desarrollo, asociados a anemia ferropriva a la edad de un año, pueden persistir a los diez años de edad y expresarse en el ámbito escolar.

(Palabras clave: anemia ferropénica del lactante, rendimiento escolar.)

\section{Referencias}

1. Rios $E$, Olivares $M$, Amar $M$, et al: Evaluation of iron status and prevalence of iton deficiency in infanıs. En: Nutrition. Intervention strategies in national development. Ed. B. Underwood. Academic Press, NY, $1983 ; 35-42$. 
2. Calvo $E$, Gnazzo $N$ : Prevalence of iron deficiency in children aged 9-24 mo from a large urban area of Argentina. Am J Clin Nucr 1990: 52: 534-540.

3. Dobbing $J$ : The later development of the brain and its vulnerability. Davis, J.A., Dobbing J.(Ed.), Scientific foundation of pediatrics, University Park/Baltimore. 1982: 744-759.

4. Waiter T, De Andraca I, Chadud P, et al: Iron deficiency anemia: Adverse effects on infant psychomotor development. J Pediatr 1989; 84: 7.17.

5. Lozoff $B$. Briffenham $G$, Wolf $A$. Iron-deficiency anemia and iron therapy: Effects on infant developmental test performance. J Pediatr 1987; 79; 981-995

6. Lozoff $B$, Brittenham $G$, Viteri $F$, et al: The effects of short term oral iron therapy on developmental deficits in iron deficient anemic infants. J Pediatr 1982: 100: 948-952.

7. Palt $H$. Pevsner B, Adler B; Does anemia in infancy affect achievement on developmental and intelligence test? Hum Biol 1985; 55: 189-194.

8. Walser $T$ : lnfancy: mental and motor developmenı. Am J Nutr 1989; 50: 655-666.

9. De Andraca l, Walter $T$, Castillo $M$, et al: Iron deficiency anemia in infancy and its effects upon psychological development at preschool age: a longitudinal study. Annual report for the study of the problem of nutrition in the world. Nestle Foundation, Laussanne, Switzerland, 1991: 53-61.

10. Lozoff $B$, Jimenez $E$, Wolf A: Long-term developmental outcome of infants with iron deficiency. $N$ Engl J Med 1991: 325: 687-694.

11. De Andraca I, Salas $l$, De la Parra $A$, et at: Interacción madre-hijo y conducta del niño en preescolares con antecedentes de anemia por deficiencia de hierto en la infancia. Arch Latinoam Nutr 1993; 43: 191-198.

12. Idjradinata $P$. Pollit $E$ : Reversal of developmental delays in iron deficient anaemic infants treated with iron. Lancet 1993; 341: 1-4.

13. Terman $L$, Merril $M$ : Medidas de la inteligencia. Método para el empleo de las pruebas de StandfordBinet. Tercera revisión, Forma L-M, Edit. EspasaCalpe S.A., Madrid, 1975: 1-505.

14. Bruininks $R$ : Bruininks-Oseretsky test of motor proficiency. American Guidance Service, Minnesota, 1972: 1-105

15. Beery $K$ : Administration, scoring and teaching manual for the developmental test of visual-motor integration. Modem curriculum Press. Cleveland, 1982: 1-97.
16. Woodcock $R$ : Woodcock Spanish psychoeducational battery. Allen. Tex: DLM Teaching resources, 1982: 1-196.

17. Piers E. Harris D: The Piers-Harris children's selfconcept scale (the way J feel my self). Counselor tests and recording, Nashville, Tenn 1969: 1-105.

18. Montenegro H: Salud mental del escolar. Estandarización del Inventario de destrezas sociales y problemas conductuales en de $T$. Achenbach en niños de 6 a 11 años. UNICEF, Santiago de Chile, 1983: I-I62.

19. De Andraca 1, Cobo $C$ Rivera $F$, et al: Evaluación de la inteligencia a través de formas cortas de] WAIS para poblaciones de nivel socioeconómico bajo. Rev Saude Publica 1993: 27: 334-339.

20. Devins $G$, Orme $C$ : Center for epidemiological studies depression scale. Kansas, 1985: 144-160.

21. Pali $H$, Meijer $A$, Adler $B$ : Learning achievement and behavior at school of anemic and non anemic infants. Early buman development 1985; 10: 217-223.

22. Webb $T$, Oski $F$ : Iron deficiency anemia and scholastic achievement in young adolescent. J Pediatr 1973; 82: 827-830

23. Pollit $E_{1}$ Leibel $R$ : Iron deficiency and behavior. J Pediatr 1976; 88: 372-381

24. Lozoff $B$, Brittenham $G$ : Behavioral aspects of iron deficiency. Progress in Hematology 1986: 14: 2353.

25. Pollit $E$, Hathirat $P$, Kotchabhakdi $N$, ef al: Iron deficiency and educational achievement in Thailand. Am J Clin Nutr 1989; 50: 35]-357.

26. Srewondo S, Husaini $M$. Pollit E: Effects of iron deficiency on attention and learning processes in preschool children: Bandung. Indonesia. Am J Clin Nutr 1989; $50: 667-674$.

27. Massare $T$ : Effects of iron deficiency on learning nutrition program. Pennsylvania State University, Human Development College. Henderson Human Development building. Liniversity park, Pennsylvania. 1981: J6: 808-81].

28. Dallman P, Simes $M$ : Brain iron: Persistent deficiency following short-tern iron deprivation in the young rat. Brit J Hemat 1975; 31: 209-215.

29. Moore $L$. Lombardi $D$, White $M$ : Influence of parent's physical activity levels on activity levels of young children. J Pediatr 1991: I I8: 215-219.

30. Lozoff $B$, Klein N, Prabuki K: Iron deficient anemic at play. Dev Behav Pediatt 1986; 7 : 152.158. 